\title{
Medikamentenpolitik der FMH
}

An einer von der FMH organisierten Tagung zum Thema Medikamentenpolitik nahmen Vertreter verschiedener Interessengruppen und Experten teil. Diskutiert wurden sowohl wissenschaftliche als auch politische Aspekte der Arzneimitteltherapie und des Arzneimittelverkaufs.

Auf dem Hintergrund der gegenwärtigen Diskussionen über die Kosten im Gesundheitswesen standen wie zu erwarten auch die Arzneimittelkosten im Zentrum des Interesses. Es wurden verschiedene Möglichkeiten diskutiert, wie im Bereich der Medikamentendistribution durch geeignete Wettbewerbsbedingungen neue Sparmöglichkeiten erschlossen werden können. Modelle aus dem In- und aus dem Ausland zeigen, dass mit neuen Anreizen der Wettbewerb gefördert und letztlich auch die Selbstverantwortung der Konsumenten gestärkt werden kann. Uneinigkeit bzw. Unsicherheit herrschte darüber, ob durch die direkte Medikamentenabgabe durch den Arzt erhöhte oder geringere Kosten entstehen. Obschon die direkte Medikamentenabgabe an sich einen Anreiz zur Mengenausweitung darstellt, ist es wahrscheinlich, dass dieser Nachteil durch den Vorteil der besseren Compliance und der günstigeren SL-Medikamente aufgewogen wird.

Gleichzeitig hat der Rückzug des Medikamentes Vioxx das Bewusstsein über die Probleme der Medikamentensicherheit wieder geschärft. Eine am Universitätsspital Zürich und am Kantonsspital St. Gallen durchgeführte und von Frau PD Dr. Karin Fattinger präsentierte Untersuchung konnte zeigen, dass bei etwa 11\% aller Patienten ein unerwünschtes Arzneimittelereignis beobachtet wurde: $4 \%$ der Patienten traten wegen eines derartigen Ereignisses ein und bei 7,5\% kam es während des Spitalaufenthaltes $\mathrm{zu}$ einem unerwünschten Arzneimittelereignis. Bei 0,21\% aller Patienten verlief das vor Eintritt bzw. während des Aufenthalts aufgetretene unerwünschte Arzneimittelereignis tödlich. Die Inzidenz von fehlerbedingten unerwünschten Arzneimittelwirkungen betrug $0,4 \%$, die Inzidenz von nicht fehlerbedingten unerwünschten Arzneimittelereignissen betrug 7,1\%. Bei den unerwünschten Arzneimittelereignissen bei Eintritt handelt es sich bei 1,1\% um fehlerbedingte unerwünschte Arzneimittelereignisse, während bei 2,9\% eine nicht fehlerbedingte unerwünschte Arzneimittelwirkung vorlag. Die Hauptursachen der Fehler waren verpasste Kontraindikationen, verpasste Interaktionen und nicht individuell angepasste Dosierungen. Diese Tatsache illustriert, dass umfassende Informationen zur aktuellen Medikation, Kontraindikationen und Begleiterkrankungen für eine individuell angepasste Pharmakotherapie essentiell sind. Entsprechend könnten Informationen zur Medikation, zu Medikamentenunverträglichkeiten und zu Begleitkrankheiten im Rahmen einer in die Krankengeschichte eingebetteten elektronischen Verschreibung viele Fehler abfangen und damit die Patientensicherheit verbessern.

Wie die Forderungen nach Kostendämpfung und erhöhter Sicherheit gleichzeitig unter einen Hut zu bringen sind, konnte keiner der anwesenden Personen aufzeigen. Immerhin schien sich ein Konsens darüber zu bilden, dass auf allen Stufen der Medikamentenpreisbildung Wettbewerbshindernisse aus dem Weg geräumt werden sollten.

Aus Sicht der FMH kann nach dieser Tagung das folgende - vorläufige - Fazit gezogen werden. 
- Der Patient soll vom Arzt über Nutzen und unerwünschte Wirkungen der Arzneimittelbehandlung aufgeklärt werden. Bei der Wahl des Arzneimittels soll auch das KostenNutzen-Verhältnis mitberücksichtigt werden.

- Der Patient (= Kunde) soll unter verschiedenen Angeboten auswählen können. Er soll selbst entscheiden, ob er seine Arzneimittel beim Arzt oder in der Apotheke kaufen will.
- Der Patient soll die Einhaltung von Qualitäts- und Sicherheitsstandards bei der Wahl und beim Verkauf des Arzneimittels selbst überprüfen können.

Dr. med. Markus Trutmann, Chefredaktor Schweizerische Ärztezeitung

Ein ausführlicher Bericht über diese Tagung folgt. 